\title{
History and perspectives of induction technology for agarwood production from cultivated Aquilaria in Asia: a review
}

\begin{abstract}
Agarwood, the resinous product of Aquilaria spp. (Thymelaeaceae), is highly valued for medicinal and fragrant purposes. Unsustainable forest harvesting contributes to the declining population in the wild, threatening their existence. As a protection effort, cultivation occurred in range countries, mainly in Asia, effectively establishing mass plantations. The success of domesticating Aquilaria relies heavily on technological advancement in agarwood induction, without which the entire thriving industry will collapse. In this paper, we describe efforts since 1929 and current progress and variation in induction as practiced in Asia. The methods include traditional practices and artificial induction via inoculum and chemical inducer, experimented and patented or otherwise. Artificial induction methods are being developed to intensify agarwood production in terms of yield and quality. While traditional methods are commonly applied by rural planters, artificial methods with the use of specific agents/chemicals are seen as more appealing for inducing agarwood, and have a higher demand from entrepreneurs and plantation owners. Several issues related to induction technology faced by stakeholders in agarwood cultivation are presented, such as safety levels, cost, yields, and quality. We conclude by highlighting remaining challenges in induction methods and their associated technologies.
\end{abstract}

Keyword: Aquilaria; Agarwood; Cultivation; 'Gaharu'; Inducer; Inoculum 